\title{
The Effect of the Presence of Lower Urinary System Symptoms on the Prognosis of COVID-19: Preliminary Results of a Prospective Study
}

\author{
Ibrahim Karabulut ${ }^{a} \quad$ Ahmet Emre Cinislioglu $^{a}$ Nazan Cinislioglu ${ }^{b}$ \\ Fatih Kursat Yilmazel ${ }^{\mathrm{a}}$ Mustafa Utlu ${ }^{\mathrm{c}}$ Handan Alay $^{\mathrm{b}}$ Erkan Cem Celik ${ }^{\mathrm{d}}$ \\ Senol Adanure \\ ${ }^{a}$ Department of Urology, Health Sciences University Regional Training and Research Hospital, Erzurum, Turkey; \\ ${ }^{b}$ Department of Infectious Diseases and Clinical Microbiology, Ataturk University Medical Faculty, Erzurum, Turkey; \\ 'Department of Internal Medicine, Health Sciences University Regional Training and Research Hospital, Erzurum, \\ Turkey; ${ }^{d}$ Department of Anesthesiology and Reanimation, Ataturk University Medical Faculty, Erzurum, Turkey; \\ e Department of Urology, Ataturk University Medical Faculty, Erzurum, Turkey
}

\section{Keywords}

COVID-19 $\cdot$ Lower urinary tract symptoms $\cdot$ International prostate symptom score $\cdot$ Benign prostatic hyperplasia

\begin{abstract}
Purpose: To investigate the effectiveness of benign prostatic hyperplasia (BPH)-related lower urinary tract symptoms (LUTS), which occur as a natural result of aging and androgen exposure, in predicting disease prognosis in male patients diagnosed with COVID-19. Methods: The study was planned prospectively. The study included 63 male patients over 40 years of age diagnosed with COVID-19. The patients were diagnosed with COVID-19 based on the results of reverse transcription polymerase chain reaction tests of oropharyngeal and nasopharyngeal swabs obtained as per the World Health Organization guidelines. The presence of LUTS was assessed by the International Prostate Symptom Score (I-PSS), a subjective assessment, and the I-PSS was filled for the patients included in the study. The patients were divided into three groups based on their scores in the I-PSS survey: group 1: mild (0-7), group 2: moderate (8-19), and group 3: severe (20-35). The data of all three groups were statistically
\end{abstract}

analyzed. Results: In the assessment performed between the groups, it was identified that for patients in group 3, the length of hospital stay was longer, intensive care requirement was more frequent, and their mortality rates were numerically higher. In the evaluation made regarding the time to intensive care admittance, this was identified to be the shortest in group 3. Conclusion: As a result of our study, we think that in patients with COVID-19, BPH-related LUTS can guide clinicians in predicting prognosis.

๑) 2020 S. Karger AG, Basel

\section{Introduction}

Lower urinary tract symptoms (LUTS) is a term that covers a variety of symptoms that occur during storing, urination, or after urination categorized by the International Continence Society [1]. LUTS are common in adult men and are often associated with benign prostatic hyperplasia (BPH) [2]. The prevalence of $\mathrm{BPH}$ increases markedly with age. In autopsy studies, it has been observed that the histological prevalence of the disease also increases with age [3]. Despite decades of intensive re- karger@karger.com

www.karger.com/uin

Karger ${ }^{\prime}=$
(C) 2020 S. Karger AG, Basel

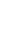

Dr. Ahmet Emre Cinislioglu

Department of Urology

Health Sciences University Regional Training and Research Hospital

TR-25040 Palandöken, Erzurum (Turkey)

emrecinisli@hotmail.com 
search, the etiology of $\mathrm{BPH}$ has still not been elucidated today. Still, the predominant hypotheses are that age-related changes and androgens play a role in the pathogenesis [4].

The coronavirus 2019 disease (COVID-19), which started in the city of Wuhan in China turned into a worldwide pandemic, infected millions of people, and caused the death of hundreds of thousands [5]. While infected individuals mostly experience mild symptoms such as fever, upper respiratory tract symptoms, shortness of breath, and diarrhea [6], or are asymptomatic [7], in severe cases, pneumonia, multiple organ failure, and death may occur [8]. The most common comorbidities in COVID-19-positive patients are chronic lung disease, diabetes and hypertension [9].

Studies conducted after the COVID-19 pandemic show that the disease is more severe and fatal in men, possibly due to the gender-based (androgens) immunological response and additional factors [10]. It has been shown that angiotensin-converting enzyme (ACE) receptors in the lung and the transmembrane serine protease 2 (TMPRSS2) enzyme group are effective in the penetration and spread of the virus into pneumocytes $[11,12]$. In recent studies, androgen-mediated regulation of the ACE receptors and the TMPRSS2 enzyme group in the host has been implicated in the more frequent occurrence of acute respiratory syndrome coronavirus-2 (SARS-CoV2) infection and higher mortality in men $[13,14]$. In this study, we aimed to investigate the effectiveness of BPH-related LUTS, which emerge as a natural result of aging and androgen exposure, in predicting disease prognosis in male patients diagnosed with COVID-19.

\section{Material and Method}

Prior to the study, approval was obtained from the local ethics committee and the Scientific Research Platform of the Ministry of Health of the Republic of Turkey. The study was planned prospectively. The study included 63 male patients over 40 years of age diagnosed with COVID-19 between April 1, 2020, and May 15, 2020. The patients were diagnosed with COVID-19 based on the results of reverse transcription polymerase chain reaction (RT PCR) tests of oropharyngeal and nasopharyngeal swabs obtained as per the World Health Organization guidelines [11]. PCR swabs were obtained using a specific swab under sterile conditions. The swab samples were sent to the Health Ministry laboratories in special containers for testing. Only the patients with positive RT PCR results were included in the study.

Patients who had a history of previous pelvic organ surgery and/or radiotherapy, who were under medical treatment (alphablocker, etc.) or had a history of surgical treatment for BPH, those with an active urinary system infection or who were under medical treatment for a disease that caused LUTS (anticholinergic, etc.), those with a history of cystoscopy for urethral stenosis, and those who had been diagnosed with neurogenic bladder, were excluded from the study.

The presence of LUTS was assessed by the International Prostate Symptom Score (I-PSS), a subjective assessment, and the I-PSS was filled for the patients included in the study. Due to the density of patients in the wards participating in this study in our pandemic hospital and the possibility of transmission to health studies, the study was limited to the I-PSS survey only. The patients were divided into three groups based on their scores in the I-PSS survey: group 1: mild (0-7), group 2: moderate (8-19), and group 3: severe (20-35). The patients' findings at presentation such as fever, cough, sore throat, headache, fatigue, muscle pain and dyspnea, and the current accompanying diseases (diabetes mellitus, hypertension, chronic lung diseases, malignancy, chronic organ failure, etc.) were recorded. In cases where intensive care hospitalizations had been necessary during the treatment period, the number of days that the patients had been hospitalized at the regular ward prior to referral to the intensive care unit and the mortality rates were recorded. The data of all three groups were statistically analyzed.

\section{Sample Size Calculation and Power Analysis}

The post hoc sample size calculation was based on the primary outcome variable, the duration of hospitalization. Calculations were made with the $G^{*}$ Power program (Heinrich-Heine-University, Düsseldorf, Germany). The effect size was calculated from our findings. Sample size calculation for a two-sided hypothesis with three groups having 22, 22 and 19 patients, consecutively I-PSS 1, I-PSS 2 and I-PSS 3 (total: 63 participants), provides a power of $82 \%$ and a Cohen's $f$ effect size of 0.41 (medium-large) to compare the three groups with one-way ANOVA. This result shows that the sample size is sufficient.

\section{Statistical Analysis}

Data analysis was carried out using the IBM SPSS 20.0 software (SPSS Inc., Chicago, IL, USA). The distribution of variables was evaluated for normality using the Shapiro-Wilk tests. The descriptive data were expressed as the mean \pm standard deviation. Categorical variables were analyzed using the Pearson $\chi^{2}$ test. The normally distributed data including continuous variables were analyzed using the Kruskal Wallis-Monte Carlo (post hoc Dunn's test). A $p$ value of $<0.05$ was considered statistically significant.

\section{Results}

The study was launched with 78 patients. After the exclusion criteria, it was completed with 63 patients (Fig. 1). Group 1 with I-PSS scores between 0-7 included 22 (34\%), group 2 with I-PSS scores between 8-19 included 22 (34\%), and group 3 with I-PSS scores between 20-35 included 19 (32\%) of the 63 patients. The characteristics of the patients are presented in Table 1. No significant difference was observed between the groups in terms of demographic findings, comorbid diseases, or presentation symptoms (Table 1). 


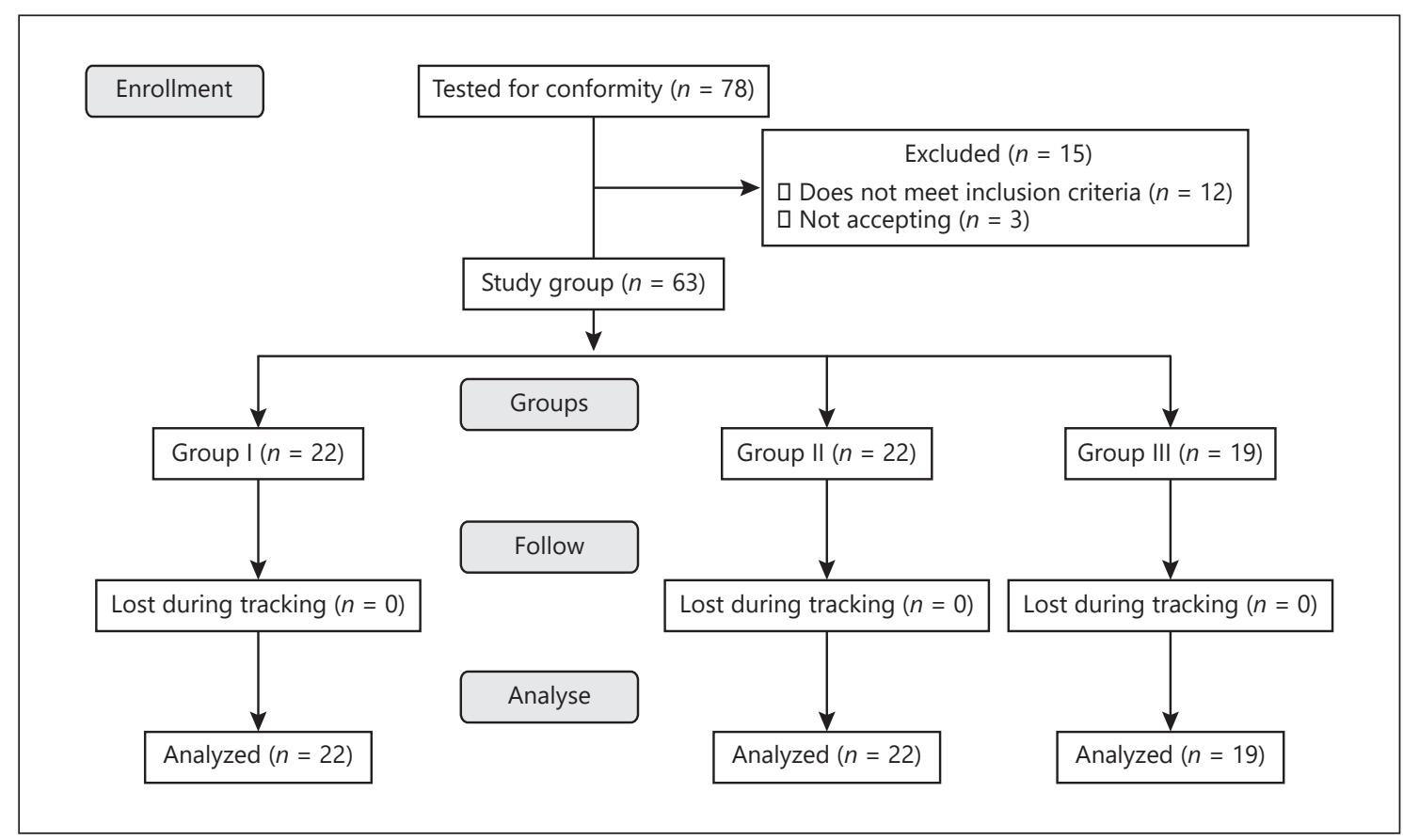

Fig. 1. Flow chart showing the number of patients included and excluded in the study.

In the assessment performed between the groups, it was identified that for patients in group 3, the length of hospital stay was longer, intensive care requirement was more frequent and their mortality rates were numerically higher. This difference was statistically significant between group 1 and group 3. The data on the length of hospital stay, intensive care requirement, and mortality rates between the groups are displayed in Table 2 .

In the evaluation made regarding the time to intensive care admittance, this was identified to be the shortest in group 3, and it was observed that this difference was statistically significant (Fig. 2; Table 3).

\section{Discussion}

Since the report of the first cases of pneumonia of unknown cause by the WHO at the end of 2019, the SARS-2 Coronavirus (Sars-CoV-2) and its related disease, COVID-19, has spread rapidly all over the globe. According to the online database of the Johns Hopkins University by April 20th, 2,411,553 infections, 165,338 deaths, and 628,816 recoveries had been confirmed worldwide [15].

Current evidence has shown that the COVID-19 mortality rate is due to comorbidities [16]. In a study con-
Table 1. Comparison of demographic findings and voiding parameters of patients in Group 1 and Group 2 before treatment

\begin{tabular}{lccl}
\hline & $\begin{array}{c}\text { Group 1 } \\
(n=34)\end{array}$ & $\begin{array}{c}\text { Group 2 } \\
(n=34)\end{array}$ & $p$ \\
\hline Age, years & $46.5 \pm 9.9$ & $43.1 \pm 7.2$ & $0.708^{\mathrm{a}}$ \\
BMI, kg/m & $24.8 \pm 2.2$ & $24.5 \pm 2.2$ & $0.896^{\mathrm{a}}$ \\
Daily urinations, $n$ & $11.2 \pm 2.1$ & $11.2 \pm 2.0$ & 0.830 \\
Urine volume, mL & $252.2 \pm 81.7$ & $261.4 \pm 94.4$ & 0.668 \\
Q $_{\text {max }}, \mathrm{mL} / \mathrm{s}$ & $11.0 \pm 2.7$ & $11.1 \pm 2.6$ & 0.964 \\
$\mathrm{Q}_{\text {mean }}, \mathrm{mL} / \mathrm{s}$ & $6.0 \pm 1.1$ & $6.0 \pm 1.1$ & 0.918 \\
PVR, mL & $83.2 \pm 25.4$ & $82.7 \pm 24.8$ & 0.942 \\
Udi 6, median score & 13.5 & 13.5 & 0.995 \\
EMG, $n(\%)$ & $34(100)$ & $34(100)$ & $1^{\mathrm{b}}$ \\
\hline
\end{tabular}

Data are presented as mean \pm SD or as stated. ${ }^{a}$ Kruskal Wallis. ${ }^{b}$ Intergroup comparison was performed using the $\chi^{2}$ test.

ducted on 45,000 Chinese COVID-19 positive patients, it was reported that while the overall mortality rate was $0.9 \%$ in patients with no comorbidity, this rate increased to $10.5,7.3$, and $6.3 \%$ in patients with cardiovascular disease, diabetes mellitus, and hypertension, respectively [17]. The outbreak in Italy started in the Lombardy region, but quickly spread to the whole country. By March 
Table 2. Comparison of the voiding parameters of patients in Group 1 and Group 2 measured before and after treatment

\begin{tabular}{|c|c|c|c|c|c|c|}
\hline & \multicolumn{3}{|c|}{ Group $1(n=34)$} & \multicolumn{3}{|c|}{ Group $2(n=34)$} \\
\hline & $\begin{array}{l}\text { before } \\
\text { treatment }\end{array}$ & 12th week & $p$ & $\begin{array}{l}\text { before } \\
\text { treatment }\end{array}$ & 12th week & $p$ \\
\hline Daily urinations, $n$ & $11.2 \pm 2.1$ & $7.7 \pm 2.9$ & $<0.001$ & $11.2 \pm 2.0$ & $9.0 \pm 3.1$ & 0.008 \\
\hline Urine volume, $\mathrm{mL}$ & $252.2 \pm 81.7$ & $261.7 \pm 77.5$ & 0.622 & $261.4 \pm 94.4$ & $258.0 \pm 82.0$ & 0.815 \\
\hline $\mathrm{Q}_{\max }, \mathrm{mL} / \mathrm{s}$ & $11.0 \pm 2.7$ & $14.8 \pm 2.6$ & 0.001 & $11.1 \pm 2.6$ & $12.8 \pm 2.9$ & 0.005 \\
\hline $\mathrm{Q}_{\text {mean }}, \mathrm{mL} / \mathrm{s}$ & $6.0 \pm 1.1$ & $8.0 \pm 1.0$ & 0.001 & $6.0 \pm 1.1$ & $6.7 \pm 1.1$ & 0.007 \\
\hline PVR, mL & $83.2 \pm 25.4$ & $54.7 \pm 31.5$ & $<0.001$ & $82.7 \pm 24.8$ & $65.7 \pm 24.1$ & 0.005 \\
\hline Udi6, score & 13.5 & 9 & $<0.001$ & 13.5 & 12 & 0.002 \\
\hline $\mathrm{EMG}, n(\%)$ & $34(100)$ & $14(41.2)$ & $0.391^{\mathrm{a}}$ & $34(100)$ & $22(64.7)$ & $0.122^{\mathrm{a}}$ \\
\hline
\end{tabular}

Data are presented as mean \pm SD or as stated. ${ }^{a}$ Intergroup comparison was performed using the $\chi^{2}$ test.

Table 3. Comparison of voiding parameters of patients in Group 1 and Group 2 at week 12

\begin{tabular}{lccl}
\hline & $\begin{array}{c}\text { Group 1 } \\
(n=34)\end{array}$ & $\begin{array}{c}\text { Group 2 } \\
(n=34)\end{array}$ & $p$ \\
\hline Daily urinations, $n$ & $7.7 \pm 2.9$ & $9.0 \pm 3.1$ & 0.078 \\
Urine volume, $\mathrm{mL}$ & $261.7 \pm 77.5$ & $258.0 \pm 82.0$ & 0.850 \\
$\mathrm{Q}_{\max }, \mathrm{mL} / \mathrm{s}$ & $14.8 \pm 2.6$ & $12.8 \pm 2.9$ & $\mathbf{0 . 0 2 6}$ \\
$\mathrm{Q}_{\text {mean }}, \mathrm{mL} / \mathrm{s}$ & $8.0 \pm 1.0$ & $6.7 \pm 1.1$ & $\mathbf{0 . 0 4 3}$ \\
PVR, $\mathrm{mL}$ & $54.7 \pm 31.5$ & $65.7 \pm 24.1$ & $\mathbf{0 . 0 2 3}$ \\
Udi6, score & 9 & 12 & $\mathbf{0 . 0 3 4}$ \\
EMG, $n(\%)$ & $14(41.2)$ & $22(64.7)$ & $\mathbf{0 . 0 0 9}$ \\
\hline
\end{tabular}

Data are presented as mean $\pm \mathrm{SD}$ or as stated. ${ }^{\text {a }}$ Intergroup comparison was performed using the $\chi^{2}$ test.

$30,2020,97,689$ confirmed cases and 10,779 deaths from the disease were reported in Italy [18]. According to the latest age-related mortality analysis published by the Italian "Istituto Superiore di Sanità" (ISS) on March 30th, $2020,8.9 \%$ of the deceased patients were over $90,39.7 \%$ were $80-89$ years of age, $35.5 \%$ were $70-79,11.2 \%$ were $60-69$, and $0.9 \%$ were $40-49$ years of age. Similar to the statistics of the research from China, this study also reported that patients with one or more comorbidity diagnosed with COVID-19 had higher mortality rates [19].

One of the most frequently reported epidemiological data is the gender-related COVID-19 mortality [19]. Studies conducted in various countries show that males are more vulnerable to COVID-19 infections, and for this reason, the male gender is considered a poor prognostic factor by some authors [20]. Male patients comprise 73\%

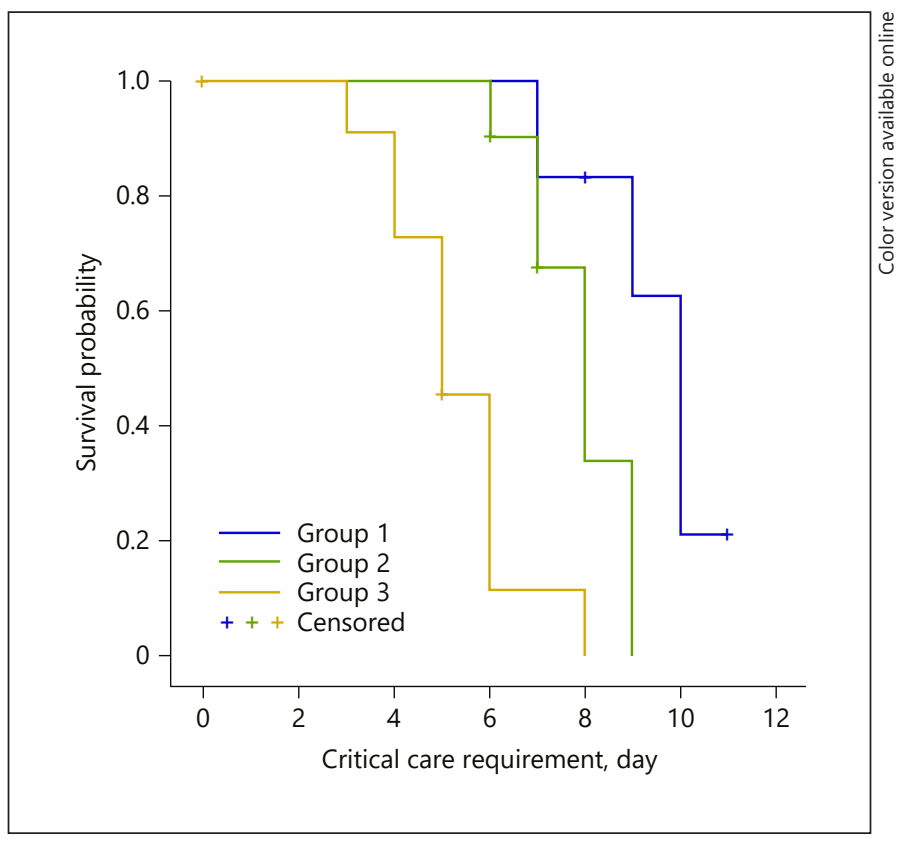

Fig. 2. Kaplan-Meier survival curves of the critical care requirement of the patients during the first 12 days $(\log \operatorname{rank} p<0.05)$.

[21] of the deaths in China, 59\% of the deaths in South Korea [22], and ISS has reported that $70 \%$ of the patients that died in Italy were male [19]. Furthermore, a recent review of current epidemiological studies that gathered data from 59,254 patients from 11 different countries has shown there is a relationship between the male gender and higher mortality rates [23]. These findings indicate there is a vulnerability regarding the male gender. The experimental study conducted by Channappanavar et al. 
[24] revealed that male rats were more vulnerable to SARS-CoV infections than age-matched female rats. Epidemiological COVID-19 studies are essential to better understand the pathogenic mechanisms of the disease and to identify superior therapeutic strategies. Although the question of why COVID-19 is more common and fatal in men remains a critical question awaiting an answer, and recent studies have suggested that one of the reasons for the increased vulnerability of men could be androgenmediated mechanisms $[13,14]$.

$\mathrm{BPH}$ is one of the common causes of LUTS in older men. Age is the main factor in the development of $\mathrm{BPH}$. The incidence of $\mathrm{BPH}$ increases from $8 \%$ in the 4 th decade to over $70 \%$ in the 7 th decade [25]. Testicular androgens are needed besides aging for the development of prostatic hyperplasia. Since the prostate gland maintains its number of androgen receptors throughout life, unlike other androgen-sensitive organs, it can respond to androgens. $\mathrm{BPH}$ does not develop in those who have been emasculated before puberty or who have genetic diseases that inhibit the effect or production of androgens. The $5 \mathrm{a}$ reductase 2 enzyme bound to the nuclear membrane converts the majority of testosterone in the prostate to dihydrotestosterone (DHT), which is the primary androgen. Although the level of testosterone in the systemic circulation decreases with aging, the level of DHT and androgen receptor function within the prostate is not affected. Although the pathophysiological mechanism of $\mathrm{BPH}$ is not fully understood, in animal and human studies, the opinion is that the factor effective in this process is the DHTsupported balance between cellular reproduction and death becoming impaired in favor of reproduction. Another contributing factor in the hyperplastic effect of androgens in the prostate is the androgen receptor activity [26].

Prostatic hyperplasia increases urethral resistance, leading to compensatory changes in bladder function. As a result of increased bladder outlet resistance, the detrusor pressure also increases for urine flow. Increased detrusor resistance also causes LUTS by affecting the bladder storage function [27]. LUTS are best assessed by approved questionnaires such as the I-PSS or the American Urology Association (AUA) symptom score [28]. In the study we conducted, we identified that the intensive care requirement and the mortality rates were significantly higher in patients diagnosed with COVID-19 with high I-PSS scores than those with low I-PSS scores. We also observed that the time to admission to intensive care was shorter in patients with high I-PSS scores compared to patients with low I-PSS scores. The data we ob- tained in this study conducted following the hypothesis that LUTS can be effective in predicting the severity of COVID-19 suggest that LUTS can be a predictor for the course and severity of COVID-19. Due to the load on the services of our pandemic hospital that participated in this study, it was limited with only the I-PSS survey. Currently, our group plans to conduct a controlled clinical trial to determine whether there is a correlation between androgens and COVID-19 severity following this preliminary observation. If LUTS caused by $\mathrm{BPH}$ were identified as a risk factor for the increased infection severity of COVID-19, we believe that these symptoms can guide physicians on the clinical course and severity of the disease. Furthermore, although no direct link has been established between the polymorphisms at the androgen receptor and COVID-19 severity as a result of our pilot study, we believe that gender inequality in the prognosis of the disease may be related to androgen receptor sensitivity or androgen exposure. We consider that 5-alpha reductase inhibitor administration alone or in combination with alpha blocker treatment would contribute to treatment of the patients who have LUTS due to BPH.

The limitations of our study were the use of I-PSS, which is only a subjective assessment of patients due to the reasons we explained in the discussion, not including a control group in the study, and the small study group.

In conclusion, in the light of the data we obtained in our study, we believe that LUTS can guide physicians in the course of COVID-19, and multicenter randomized prospective studies are needed to determine the potential relationship between androgens and COVID-19 severity.

\section{Statement of Ethics}

Prior to this single-center prospective study, approval was obtained from the Ethics Committee of Atatürk University Faculty of Medicine (approval No.: B.30.2.ATA.0.01.00/322). This study was conducted as per the latest version of the Helsinki Declaration and the Guidelines for Good Clinical Practice. Informed consent was obtained from all patients who participated in the study.

\section{Conflict of Interest Statement}

There is no conflict of interest of any authors in relation to the submission. 


\section{Funding Sources}

This research received no specific grant from any funding agency in the public, commercial, or not-for-profit sectors.

\section{Author Contributions}

Ibrahim Karabulut: writer, design, analysis; Ahmet Emre Cinislioglu: supervision, design; Nazan Cinislioglu: design; Hadan Alay: data collection, design; Fatih Kursat Yilmazel: data collection, design; Mustafa Utlu: data collection, design; Erkan Cem Celik: analysis; Senol Adanur: design.

\section{References}

1 Abrams P, Cardozo L, Fall M, Griffiths D, Rosier P, Ulmsten U, et al.; Standardisation Sub-committee of the International Continence Society. The standardisation of terminology of lower urinary tract function: report from the Standardisation Sub-committee of the International Continence Society. Neurourol Urodyn. 2002;21(2):167-78.

2 McNicholas T, Kirby R. Benign prostatic hyperplasia and male lower urinary tract symptoms. Am Fam Physician. 2012 Aug 15;86(4): 359-360.

3 Langan RC. Benign Prostatic Hyperplasia. Prim Care. 2019 Jun;46(2):223-32.

4 Gandaglia G, Briganti A, Gontero P, Mondaini N, Novara G, Salonia A, et al. The role of chronic prostatic inflammation in the pathogenesis and progression of benign prostatic hyperplasia (BPH). BJU Int. 2013 Aug; 112(4):432-41.

5 Ge H, Wang X, Yuan X, Xiao G, Wang C, Deng $\mathrm{T}$, et al. The epidemiology and clinical information about COVID-19. Eur J Clin Microbiol Infect Dis. 2020 Jun;39(6):1011-9.

6 Guan WJ, Ni ZY, Hu Y, Liang WH, Ou CQ, He JX, et al.; China Medical Treatment Expert Group for Covid-19. Clinical Characteristics of Coronavirus Disease 2019 in China. N Engl J Med. 2020 Apr;382(18):1708-20.

7 Bai Y, Yao L, Wei T, Tian F, Jin DY, Chen L, et al. Presumed Asymptomatic Carrier Transmission of COVID-19. JAMA. 2020 Feb; 323(14):1406-7.

8 Weiss P, Murdoch DR. Clinical course and mortality risk of severe COVID-19. Lancet. 2020 Mar;395(10229):1014-5.

9 Cheng VC, Wong SC, Chen JH, Yip CC, Chuang VW, Tsang OT, et al. Escalating infection control response to the rapidly evolving epidemiology of the coronavirus disease 2019 (COVID-19) due to SARS-CoV-2 in Hong Kong. Infect Control Hosp Epidemiol. 2020 May;41(5):493-8.

10 Chen N, Zhou M, Dong X, Qu J, Gong F, Han $\mathrm{Y}$, et al. Epidemiological and clinical characteristics of 99 cases of 2019 novel coronavirus pneumonia in Wuhan, China: a descriptive study. Lancet. $2020 \mathrm{Feb}$;395(10223):507-13.

11 Leung JM, Yang CX, Tam A, Shaipanich T, Hackett TL, Singhera GK, et al. ACE-2 expression in the small airway epithelia of smokers and COPD patients: implications for COVID-19. Eur Respir J. 2020 May;55(5): 2000688.
12 Stopsack KH, Mucci LA, Antonarakis ES, Nelson PS, Kantoff PW. TMPRSS2 and COVID-19: Serendipity or Opportunity for Intervention? Cancer Discov. 2020 Jun;10(6): 779-82.

13 Wambier CG, Goren A. Severe acute respiratory syndrome coronavirus 2 (SARS-CoV-2) infection is likely to be androgen mediated. J Am Acad Dermatol. 2020 Jul;83(1):308-9.

14 Pozzilli P, Lenzi A. Commentary: Testosterone, a key hormone in the context of COVID-19 pandemic. Metabolism. 2020 Jul;108: 154252.

15 Johns Hopkins Coronavirus Resource Center. https://coronavirus.jhu.edu/map.html [accessed March 30, 2020].

16 Rodriguez-Morales AJ, Cardona-Ospina JA, Gutiérrez-Ocampo E, Villamizar-Peña R, Holguin-Rivera Y, Escalera-Antezana JP, et al.; Latin American Network of Coronavirus Disease 2019-COVID-19 Research (LANCOVID-19). Electronic address: https://www. lancovid.org. Clinical, laboratory and imaging features of COVID-19: A systematic review and meta-analysis. Travel Med Infect Dis. 2020 Mar - Apr;34:101623.

17 Epidemiology Working Group for NCIP Epidemic Response, Chinese Center for Disease Control and Prevention. The epidemiological characteristics of an outbreak of 2019 novel coronavirus diseases (COVID-19) in China. Zhonghua Liu Xing Bing Xue Za Zhi. 2020; 41(2):145-51.

18 Porreca A, Colicchia M, D'Agostino D, Amenta M, Corsaro A, Zaramella S, et al. Urology in the Time of Coronavirus: Reduced Access to Urgent and Emergent Urological Care during the Coronavirus Disease 2019 Outbreak in Italy. Urol Int. 2020;104(7-8): 631-6.

19 La Vignera S, Cannarella R, Condorelli RA, Torre F, Aversa A, Calogero AE. Sex-Specific SARS-CoV-2 Mortality: Among HormoneModulated ACE2 Expression, Risk of Venous Thromboembolism and Hypovitaminosis D. Int J Mol Sci. 2020 Apr;21(8):E2948.
20 Mo P, Xing Y, Xiao Y, Deng L, Zhao Q, Wang $\mathrm{H}$, et al. Clinical characteristics of refractory COVID-19 pneumonia in Wuhan, China. Clin Infect Dis. 2020 Mar 16;ciaa270. doi: 10.1093/cid/ciaa270. Online ahead of print.

21 Chen $\mathrm{T}, \mathrm{Wu} \mathrm{D}$, Chen $\mathrm{H}$, Yan $\mathrm{W}$, Yang $\mathrm{D}$, Chen G, et al. Clinical characteristics of 113 deceased patients with coronavirus disease 2019: retrospective study. BMJ. 2020 Mar; 368:m1091.

22 Korean Society of Infectious DiseasesKorean Society of Pediatric Infectious DiseasesKorean Society of EpidemiologyKorean Society for Antimicrobial TherapyKorean Society for Healthcare-associated Infection Control and PreventionKorea Centers for Disease Control and Prevention. Report on the Epidemiological Features of Coronavirus Disease 2019 (COVID-19) Outbreak in the Republic of Korea from January 19 to March 2, 2020. J Korean Med Sci. 2020 Mar;35(10):e112.

23 Borges do Nascimento IJ, Cacic N, Abdulazeem HM, von Groote TC, Jayarajah U, Weerasekara I, et al. Novel Coronavirus Infection (COVID-19) in Humans: A Scoping Review and Meta-Analysis. J Clin Med. 2020 Mar;9(4):E941.

24 Channappanavar R, Fett C, Mack M, Ten Eyck PP, Meyerholz DK, Perlman S. SexBased Differences in Susceptibility to Severe Acute Respiratory Syndrome Coronavirus Infection. J Immunol. 2017 May;198(10): 4046-53.

25 Partin AW, Oesterling JE, Epstein JI, Horton $\mathrm{R}, \mathrm{Walsh}$ PC. Influence of age and endocrine factors on the volume of benign prostatic hyperplasia. J Urol. 1991 Feb;145(2):405-9.

26 Wen S, Chang HC, Tian J, Shang Z, Niu Y, Chang C. Stromal androgen receptor roles in the development of normal prostate, benign prostate hyperplasia, and prostate cancer. Am J Pathol. 2015 Feb;185(2):293-301.

27 Chughtai B, Forde JC, Thomas DD, Laor L, Hossack T, Woo HH, et al. Benign prostatic hyperplasia. Nat Rev Dis Primers. 2016;2: 16031.

28 Karavitakis M, Kyriazis I, Omar MI, Gravas S, Cornu JN, Drake MJ, et al. Management of Urinary Retention in Patients with Benign Prostatic Obstruction: A Systematic Review and Meta-analysis. Eur Urol. 2019 May;75(5): $788-98$. 